\title{
Nonlinear optical imaging techniques (NLO) for painting investigation
}

\author{
Alice Dal Fovo ${ }^{\star}$, Raffaella Fontana, Jana Striova, Enrico Pampaloni, Marco Barucci, \\ Marco Raffaelli, Raffaella Mercatelli, Luca Pezzati, Riccardo Cicchi
}

Istituto Nazionale di Ottica - CNR-INO, Largo Fermi 6, 50125, Firenze, Italy

* Corresponding author: alice.dalfovo@ino.it

\begin{abstract}
Nonlinear optical imaging techniques (NLO imaging), i.e. multiphoton excitation fluorescence (MPEF), second and third harmonic generation (SHG, THG), are high-resolution imaging modalities which may provide non-destructive determination of thickness and composition within multi-layer objects as a function of depth. NLO techniques have been recently introduced into artworks analyses, giving promising results. As regards paintings, the guiding idea is to exploit the low absorption in the near-infrared (NIR) of most materials, using a laser light in this spectral region which may generate non-linear interactions within materials. The objective is to make feasible the in-depth profiling of thin films on the basis of refractive index changes, variation of optical activity and presence of fluorophores. Such information is definitely useful for the analysis of painted objects and can be also crucial for the monitoring of restoring operations. In this study, we present the preliminary results obtained through the application of NLO imaging techniques on a series of single- and multi-layers systems simulating real egg-tempera wood panel paintings. This research is part of the wider Iperion $\mathrm{CH}$ Project, whose WP6 entails the evaluation of NLO-imaging to obtain highly resolved en-face and 3D images of small areas on paintings. To that purpose, a set of multi-layer painted samples, simulating real paintings, was designed and realized. They are currently under analysis with different operating systems and modalities within the partnership. In order to assess the reliability of NLO measurements, data were complemented with other well-established techniques, such as Fourier Transform Infrared Spectroscopy (FTIR) and X-Ray Fluorescence (XRF) for the chemical characterization of materials, Optical Coherence Tomography (OCT) for the determination of layers thickness and laser scanning micro-profilometry for the study of surface morphology. In addition, colorimetric analyses, i.e. reflectance spectroscopy in the visible region, were performed in order to chromatically characterize pigments and lakes. Finally, the transparency of the different materials was investigated by means of Scanning Multispectral VIS-NIR Reflectography.

The preliminary results suggest that the $740 \mathrm{~nm}$ wavelength, while enabling the visualization of the painting surfaces micromorphology, is not effective for the determination of thickness through the detection of the non-linear signals.
\end{abstract}

Keywords: non-invasive techniques, non-linear imaging, multi-photon excitation fluorescence, paintings, monitoring 


\section{Introduction}

Multiphoton excitation fluorescence (MPEF), second and third harmonic generation (SHG, THG), and fluorescence lifetime imaging microscopy (FLIM) are high-resolution non-linear imaging modalities, which may provide non-destructive determination of thickness and composition within multi-layer objects. NLO techniques are widely used as diagnostic tools in the biological field and have been applied to artworks analyses for the identification of layers corrosion in metal-based objects [1], for the visualization and characterization of wood microstructures [2] and for the study of synthetic glue and varnish protective layers [3-5]. In regard to paintings, only few tests have been performed by means of femtosecond pumpprobe microscopy on painted mock-ups and historic artworks [6, 7], encouraging further investigations. The guideline of this research is to examine inner layers by exploiting the low absorption of most painting materials in the near-infrared region, thus obtaining the in-depth profiling of thin films, the micrometric surface mapping and the detection of fluorescence chromophores and specific molecular species. Such information may turn useful for the analysis of painted objects, as well as for the monitoring of restoring operations, like the cleaning process, that modify surfaces micrometric morphology and coating layers thickness.

NLO techniques are based on physical processes in which a molecule can be excited by simultaneous interaction with two or more photons in the same quantum event. SHG and THG are second and third order non-linear scattering phenomena in which two or three photons of $\omega$ angular frequency are simultaneously converted in a photon of $2 \omega$ or $3 \omega$ angular frequency, respectively. SHG enables the detection of high degree-organized structures without inversion symmetry, such as stacked membranes and arranged proteins (e.g. collagens), for the visualisation of wood microstructure, specifically crystalline cellulose $[2,3]$. On the other hand, THG allows to resolve transparent interfaces, on the basis of local differences in refractive index and dispersion [8]. In Two-Photon-Excited Fluorescence (TPEF) the detection of fluorescence emitted after the simultaneous absorption of two infrared photons [9] yields to the identification of chromophores. Finally, fluorescence lifetime imaging microscopy (FLIM) measures time-decay of the fluorescence intensity exhibited by fluorescent emitting molecules [10], yielding information on the molecular microenvironment of a molecule and on its energy exchanges.

The use of non-linear techniques offers several advantages to the three-dimensional imaging, mainly because of the non-linear properties of the high-intensity excitation source employed - i.e. a tightly focused femtosecond (fs) pulsed laser. Firstly, non-linear interaction occurs only at, or near, the focal point of the beam. Secondly, long wavelengths, particularly in the near-infrared spectral region, allow deeper penetration in most pictorial materials and are not scattered as much as shorter wavelengths. Thirdly, since in non-linear processes the excitation volume is proportional to a power of the laser intensity, the axial resolution achievable is greatly increased. Finally, photo-damage and photo-bleaching are drastically reduced with respect to confocal and wide-field fluorescence imaging, which is desirable in $\mathrm{CH}$ studies.

In this work we present the preliminary results obtained through the application of NLO imaging techniques with a custom-made laser-scanning non-linear micro- 
scope developed by INO-CNR (Cicchi R. et al.) [10]. The analyses were performed on a series of single- and multi-layers systems, specifically designed for NLO imaging applications, simulating the real egg-tempera wood panel paintings. Such samples are distributed in a round robin approach within the partnership and are currently under analysis with different operating systems and modalities. This research is part of the wider IperionCH Project whose WP6 entails the evaluation of NLO-imaging to obtain highly resolved en-face and 3D images .

\section{Materials and Methods}

\subsection{Description of samples}

The samples analyzed in this study were designed to create favourable and variable condition to detect NLO signals in order to assess the applicability and the efficiency of non-linear microscopy techniques on a given number of cases. In this section, the design of the samples is reported firstly, followed by the description of material used.

Each sample is a multi-layer system simulating a real painting, showing a sequence of layers of different thickness, obtained by increasing the number of the superimposed stratifications.

We prepared three sets of samples - red, yellow and blue - labelled R, Y and B, respectively (Fig. 1). For each colour three versions, $\mathrm{A}, \mathrm{B}$ and $\mathrm{C}$, were realized, showing the same sequence of stratification and materials but different layers' thickness. Each set (R, Y, B) is composed of 24 square samples $(3 \mathrm{~cm} \times$ $3 \mathrm{~cm}$ ), for a total number of 72 squares.

The stratifications sequence is the following: wooden support (a "sandwich" structure made of fir and poplar), preparation layer (gypsum and animal glue), underdrawings (lead and tin stylus, natural carbon, iron-gall
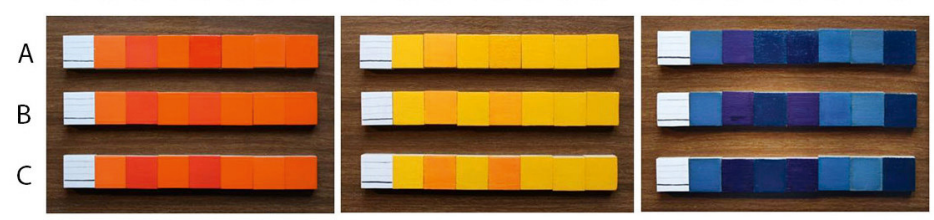

\begin{tabular}{lccl}
\hline $\begin{array}{l}\text { Colour } \\
\text { sets }\end{array}$ & Versions & $\begin{array}{c}\text { Number of square } \\
\text { samples }\end{array}$ & Acronym \\
\hline \multirow{3}{*}{ Red (R) } & A & 8 & R_A1, A2, A3, A4, A5, A6, A7, A8 \\
\cline { 2 - 4 } & B & 8 & R_B1, B2, B3, B4, B5, B6, B7, B8 \\
\cline { 2 - 4 } & C & 8 & R_C1, C2, C3, C4, C5, C6, C7, C8 \\
\hline \multirow{3}{*}{ Yellow (Y) } & A & 8 & Y_A1, A2, A3, A4, A5, A6, A7, A8 \\
\cline { 2 - 4 } & B & 8 & Y_B1, B2, B3, B4, B5, B6, B7, B8 \\
\cline { 2 - 4 } & C & 8 & Y_C1, C2, C3, C4, C5, C6, C7, C8 \\
\hline \multirow{3}{*}{ Blue (B) } & A & 8 & B_A1, A2, A3, A4, A5, A6, A7, A8 \\
\cline { 2 - 4 } & B & 8 & B_B1, B2, B3, B4, B5, B6, B7, B8 \\
\cline { 2 - 4 } & C & 8 & B_C1, C2, C3, C4, C5, C6, C7, C8 \\
\hline
\end{tabular}

Fig. 1. Multi-layers samples simulating real paintings with the relative acronyms. 
ink), first pictorial layer (egg tempera with three different pigments), second pictorial layer (three different lakes) and superficial protective layers (natural and synthetic varnishes, egg, and multi-layer coatings). The stratification sequence for version A - sets R, $\mathrm{Y}, \mathrm{B}$ - which is the first that has been analyzed for this study, is shown in Fig. 2 and described in the following scheme:

We chose the wood as a support because, besides being historically and physically suitable for egg-tempera, it also gives the opportunity to visualize wood microstructures by means of NLO signals, specifically crystalline cellulose (SHG signal) and lignin (2PEF signal) in the wood cell walls. binders, such as linseed oil). We chose three different pigments by Kremer (Germany) - i.e. lead minium (red lead, $\mathrm{Pb}_{3} \mathrm{O}_{4}$ ) - cod. 42500; cadmium yellow medium (Cadmium sulfide, CdS) - cod. 21051; Egyptian blue (blue copper silicate chemically classified as the mineral cuprorivaite, $\mathrm{CaCuSi}_{4} \mathrm{O}_{10}$ ) - cod. 10060 - in order to reproduce the optical properties of three representative painting layers. The selected pigments were widely used throughout history and show some specific optical properties, which hopefully make the study of their non-linear response relevant.

Minium is an oxidation product of lead minerals, originally extracted in the river

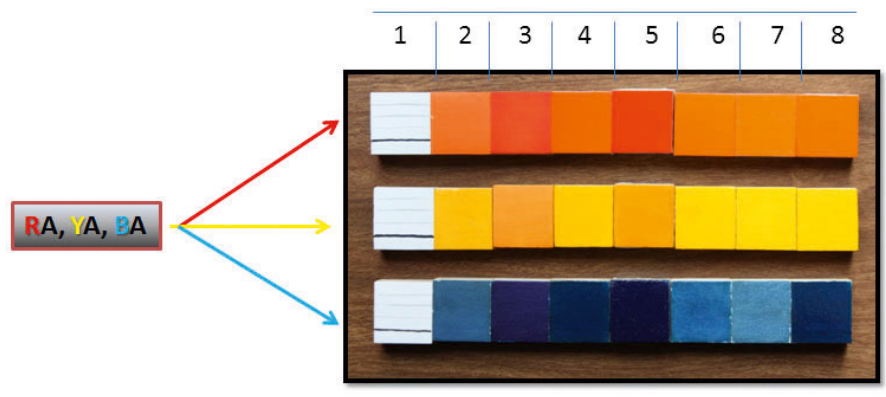

Fig. 2. Version A (basic layers thickness) of R, Y, B sets.

Scheme of the stratifications:

- 1: preparation + underdrawings

- 2: preparation + underdrawings + egg-tempera

- 3: preparation + underdrawings + egg-tempera + lake

- 4: preparation + underdrawings + egg-tempera + varnish (dammar)

- 5: preparation + underdrawings + egg-tempera + lake + varnish (dammar)

- 6: preparation + underdrawings + egg-tempera + varnish (synthetic varnish)

- 7: preparation + underdrawings + egg-tempera + protein layer (white egg)

- 8: preparation + underdrawings + egg-tempera + multi-layer coating (white egg + dammar)

The presence of the underdrawings on the preparation layer enables the evaluation of the layers' transparency. We selected the egg as a binder because of its drying speed, good stability, compatibility with most pigments, and reduced fluorescence (compared to oily
Minius in North-Western Spain, anciently produced by smelting lead ore and later prepared by heating lead white (basic lead carbonate) in air. Nowadays, it is obtained by heating litharge or white lead for some hours at high temperature (about $480^{\circ} \mathrm{C}$ ). Minium 
is an intense orange red pigment compatible with oil and egg binders, quite unstable and liable to discoloration in the presence of air pollutants, such as hydrogen sulfide, and may darken when exposed to humidity and light. Such a behaviour is limited when the pigment is dispersed in oil medium. However, given the high level of toxicity of red lead, minium has been gradually removed from the market and replaced with others, which are safer.

Cadmium sulfide refers to a number of yellow-orange-red compounds sharing a common structure based on CdS. By substituting $\mathrm{Cd}$ or $\mathrm{S}$ with various elements including $\mathrm{Zn}, \mathrm{Hg}$, and $\mathrm{Se}$, a variety of hues can be obtained. Such chromatic properties are due to $\mathrm{CdS} 2.42 \mathrm{eV}$ band gap that can be modulated by the progressive substitution of Cd with one of the former elements (e.g. Zn to obtain pale yellow cadmium zinc sulfide pigments [11]). Cadmium yellow, cadmium red and cadmium orange are brilliant pigments with good permanence and tinting power, which have been widely used since 1840, especially by painters like Claude Monet, Vincent Van Gogh, Edvard Munch and Jackson Pollock. Initially, Cadmium yellow (Cadmium sulfide, CdS) was prepared with an acid solution of cadmium salt (either chloride or sulfate) which was heated with hydrogen sulfide gas until a powder was generated to obtain hues ranging from lemon yellow to deep orange. In the past few years, once Cadmium's toxicity had been determined, the production of Cadmium pigments gradually decreased and they were partially replaced by azo-pigments, which have the advantage of being both cheaper and non-toxic. Cadmium sulfide is not very toxic either (median lethal dose $\mathrm{LD}_{50}>5000 \mathrm{mg}$ / $\mathrm{kg}$ ) when used as a pigment, although intense exposure to cadmium vapours from welding is harmful. Since Cadmium yellow optical behaviour has been thoroughly researched, we presumed it would have been significant for NLO investigations.

Egyptian blue is a copper silicate chemically classified as the mineral cuprorivaite $\left(\mathrm{CaCuSi}{ }_{4} \mathrm{O}_{10}\right)$, rarely found in nature but synthetically obtained since ancient times. Indeed, it is the first synthetic pigment that we know of, having been produced since the $4^{\text {th }}$ Egyptian Dynasty (around 2500 BC). Thanks to its chemical composition, Egyptian blue is stable in all media and does not undergo any colour change when applied to various organic media. Strong light does not influence its colour either, as it may be observed in samples which have survived for millennia in both dry and damp environments and still exhibit an intense hue with no evidence of photobleaching. Recently, a further remarkable property of this pigment has been found, namely the exceptionally high level of emission quantum efficiency in the near infrared $\left(\lambda_{\max }=\right.$ $\left.910 \mathrm{~nm} ; \Phi_{\mathrm{EM}}=10.5 \%\right)$ and the long excited lifetime $(107 \mu \mathrm{s})$, which makes cuprorivaite an excellent candidate as a luminescent label for a number of applications [12]. In view of this, we decided to investigate Egyptian blue non-linear behaviour.

A few samples (numbers 3 and 5 of each set) present an additional pictorial layer over the egg tempera, made of three different lakes, Old Holland Classic Oil Colors by Kremer (Ruby lake - R set -, Quinacridon-Anthanthrone, colour index: PR209-PR168; Blue lake - B set -, Phthalocyanine, colour index: PB15; Indian yellow-orange lake extra - Y set -, Azo condensation-Diketo Pyrrolo Pyrrole, colour index: PY95-PR251), in order to simulate real restoring interventions, and more specifically the pictorial retouching through the superposition of layers. Some other samples (numbers $4,5,6,7,8$ ) are covered 
with several protective layers: natural resin (dammar, by Zecchi) or synthetic varnish (picture varnish gloss, by Zecchi), protein white egg - layer, and a multilayer coating (white egg + dammar), so to replicate real cases of varnished paintings. The variability of paint and protective layers thickness and composition was chosen/established in order to evaluate the NLO-signals as a function of depth and of chemical composition.

\subsection{Measurements}

NLO measurements were performed using a custom-made laser-scanning nonlinear microscope, originally developed by Cicchi R. et al. for biological applications at INO-CNR [10]. The excitation source is a mode-locked Ti:Sapphire laser (Mira 900 F, Coherent Inc., Santa Clara, CA, US). The beam is focused onto the specimen by an objective (Nikon Plan Apo 20x, NA 0.75 Air). The filter used for the SHG detection is a narrow bandpass filter centred at $370 \pm 10 \mathrm{~nm}$ HQ420BP (Chroma Technology Corporation). For this study, the excitation wavelength was set to $740 \mathrm{~nm}$, with a pulse duration of $120 \mathrm{fs}$ and a repetition rate of $80 \mathrm{MHz}$, with a power of about $5 \mathrm{~mW}$ on the specimen. Non-linear signals coming from the sample were revealed by two different detection systems (Fig. 3-4): one for intensity images and the other for lifetime images. Two-photon-fluorescence intensity images are provided using a photomultiplier tube (Hamamatsu H7422), with a 190-900 nm spectral sensitivity, by assigning to each pixel an intensity value (grey level), that is proportional to the amount of fluorescence photons reaching the detector from each surface point scanned by the laser. TPEF images were acquired with a field of view of $200 \times 200 \mu^{2}$, a resolution of $512 \times 512$ pixels, z-step of $2 \mu \mathrm{m}$ for an

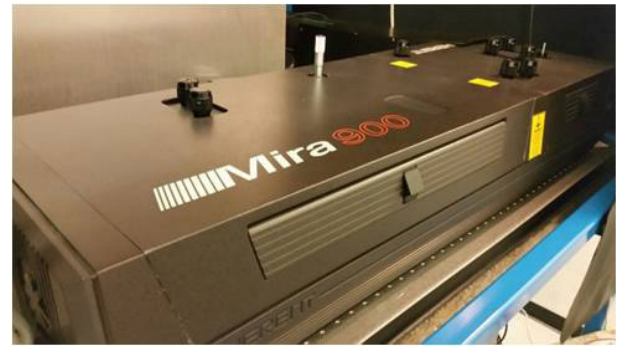

Fig. 3. Laser excitation source Mira 900 F, Coherent Inc., Santa Clara, CA, US.

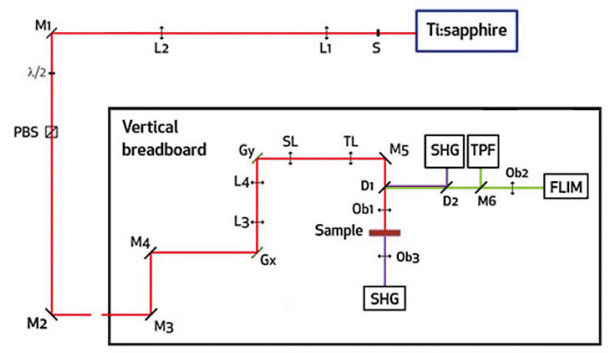

Fig. 4. Scheme of the experimental setup of the laser-scanner nonlinear microscope: laser source (Ti:Sapphire), optics (L 1-4, lens; TL, tube lens; SL, scanning lens; M 1-6, mirrors; Ob 1-3, shutters; Gx, Gy, galvanometric mirrors) and detection system (SHG, TPF, FLIM detectors).

acquisition time of about $5 \mathrm{sec}$. Using ImageJ (National Institutes of Health, Bethesda, VA, US) “Orthogonal Views" plug-in, 3D reconstructions from the stacks were obtained.

Fluorescence lifetime imaging microscopy (FLIM) was performed using a fast photon-counting detector (Becker \& Hickl PMH-100), with 400-600 nm spectral sensitivity. The output data, i.e. the decay curves and the related FLIM images, are reconstructed pixel-by-pixel from individual time measurements. Based on Time-Correlated Single-Photon Counting (TCSPC) principle, the arrival time of the individual photons is recorded with respect to the excitation pulse. Data processing and display are performed 
by means of SPC-Image software (Becker \& Hickl GmbH, Germany). The SPC-Image is coded in a false-colour scale image, in which each point represents the average fluorescence lifetime calculated through mono, bi- or tri-exponential fit of the related fluorescence decay curve, which can be visualized positioning the cursor in points of interest on the image.

Finally, a fast 16-channel photon counting photomultiplier (PML-16C Becker \& Hickl $\mathrm{GmbH}$ ) provides 16 fluorescence decay curves at 16 different narrow spectral bands for each point of the FLIM image. In this mode, the emitted light is sent to a spectrograph, it reaches the 16-channel photomultiplier with a transit time spread typical value of $150 \mathrm{ps}$, and then it is processed by the FLIM (Fluorescence Lifetime Imaging Microscopy) apparatus. Therefore, for each point of the measured area, 16 FLIM images are provided, containing the xy maps of the fluorescence decay, which are recorded with a spectral resolution of $12,5 \mathrm{~nm}$ within 400-600 $\mathrm{nm}$ instrument's operating range, thus enabling the detection of specific emitting molecular species.

In order to assess the reliability of NLO measurements, in cooperation with Opificio delle Pietre Dure (OPD- Scientific Laboratory), UV-Vis cross-section images on micro-samples taken from the edge of each sample were obtained and they were used as a reference for thickness measurements, except in cases where the polyester resin used for embedding the samples was found to dissolve the protective layers (fresh dammar or synthetic resin). Besides that, in some cases the infiltration of the polyester through the superficial porosity perceptibly increased the layer's thickness, thus affecting again the thickness evaluation. X-Ray Fluorescence (XRF) was also performed in order to chemically characterize the pigments (OPD).

Data were also complemented with well-established non-invasive techniques, such as Optical Coherence Tomography (OCT) $[13,14]$ for the determination of layers thickness, and laser scanning micro-profilometry for the study of surface morphology [15-17].

As regards OCT measurements, two different devices were used: the first one, a Time-Domain confocal OCT prototype (INO-CNR) [18], with operating wavelength $1550 \mathrm{~nm}$, which combines confocal microscope optics with the OCT technique setup (axial resolution: $10 \mu \mathrm{m}$, lateral resolution: 2,5 $\mu \mathrm{m}$ ); the second one, a Thorlabs Ganymede Series Spectral-Domain OCT System, with operating wavelength $900 \mathrm{~nm}$ (axial resolution: $3 \mu \mathrm{m}$, imaging depth: 1,9 $\mathrm{mm})$. As the tomographic images acquired with SD-OCT Thorlabs @900 nm were affected by a strong surface scattering, the interference signal turned out to be too weak to enable the layers' thickness evaluation. For that reason, those OCT images are not reported, whereas the images obtained with the Td-OCT prototype are discussed in the results section.

The microprofilometer $(1 \mu \mathrm{m}$ axial resolution, $20 \mu \mathrm{m}$ lateral resolution, $8 \mathrm{~mm}$ dynamic range) produces a topographic map of the measured surface that is subsequently processed by means of purposely developed software and displayed as simulated raking light image.

The optical behaviour of pigments and lakes in VIS and NIR regions was examined by applying colorimetric analyses and Fibre Optics Reflectance Spectroscopy (FORS). Colorimetric analysis and reflectance spectrometry in the visible range were performed using a CM-2500c Konica Minolta 
Spectrophotometer, with $45^{\circ} / 0^{\circ}$ illumination/ observation configuration, $360 \mathrm{~nm}$ to 740 $\mathrm{nm}$ spectral sensitivity, and $10 \mathrm{~nm}$ spectral resolution. Three acquisitions were made for each sampled point, calculating both their average and the relative standard deviations. Fibre optics reflectance spectra were acquired with the Spectroanalyser Carl Zeiss UV-Vis-NIR (acquisition range between 304 and $1700 \mathrm{~nm}$ ). The final spectra were obtained as an average of 3 acquisitions for each measurement. Finally, the transparency of the different materials was investigated by means of Scanning Multispectral VISNIR Reflectography whose output is a set of aberration-free monochromatic images (16 Vis + 16 NIR images; spatial sampling $250 \mu \mathrm{m}$; spectral resolution $20-30 \mathrm{~nm}$ in the Vis, and $50-100 \mathrm{~nm}$ in the NIR) in the range 380-2500 nm [19-21].

\section{Results and discussion}

The first aim of the study was to verify the capability to assess the layers' thickness through NLO signals. We started by evaluating the thickness of the egg-tempera layer for the red, yellow, blue pigments. It is worth underlining that the results presented here are preliminary and request deeper investigations.

Measurements were initially conducted on samples R_A2,Y_A2, B_A2 (egg tempera without superficial varnish, Fig. 5, 6, 7) in order to collect only non-linear signals coming from the painting with no interference due to the fluorescence of varnishes.

The painted layers thickness estimated through cross section analysis is consistent with that obtained with OCT measurements. In specific, the red-tempera layer thickness resulted between 35 and $45 \mu \mathrm{m}$ (Fig. 8, 9); the yellow tempera $45-55 \mu \mathrm{m}$ (Fig. 10, 11); the blue tempera $120-150 \mu \mathrm{m}$ (Fig. 12, 13).

With regard to these results, the layers' thickness measured through NLO proves to be considerably underestimated in all cases: the results provided by TPEF imaging were around $5 \mu \mathrm{m}$ for the red sample (Fig. 14), 4 $\mu \mathrm{m}$ for the yellow sample (Fig. 15) and $10 \mu \mathrm{m}$ for the blue sample (Fig. 16). Even with the increase of the beam power, no deeper detection was obtained. The fact that no signal over $15 \mu \mathrm{m}$ depth was detected by NLO, even if the two-photon excitation fluorescence is very likely to occur at greater depth, can be explained by the following hypothesis: the $740 \mathrm{~nm}$ excitation wavelength used for NLO measurements, while enabling an adequate penetration inside the material, generates TPEF signals which are likely to undergo absorption and attenuation effects before reaching the external surface.

To support this explanation, the optical properties of the three pigments have been compared with the spectra obtained by FORS and the multispectral reflectance images acquired on samples R2, Y2, B2 in the spectral range of interest (Fig. 17).

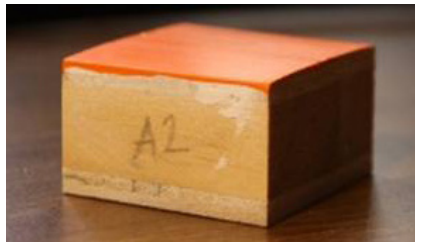

Fig. 5. Sample R_A2.

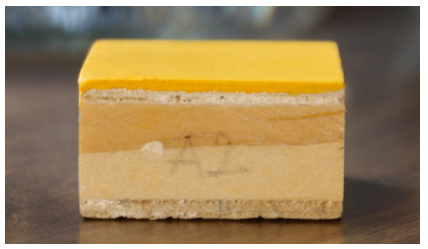

Fig. 6. Sample Y_A2.

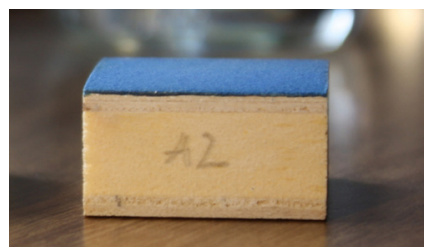

Fig. 7. Sample B_A2. 


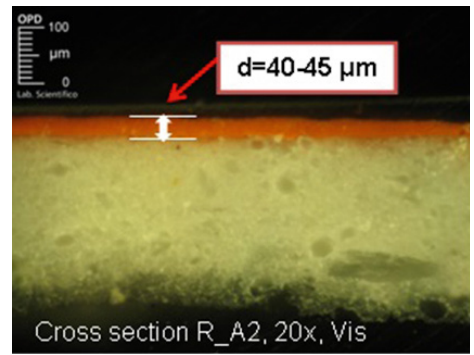

Fig. 8. Cross section R_A2, 20x, Vis (OPD).

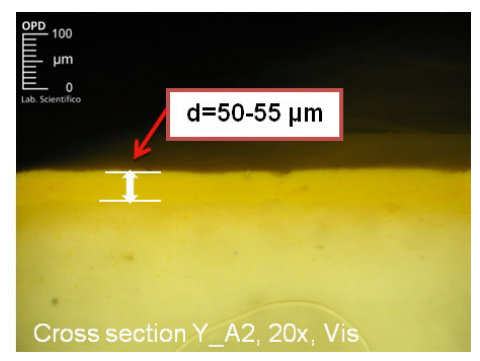

Fig. 10. Cross section $Y_{-}$A2, 20x, Vis (OPD).

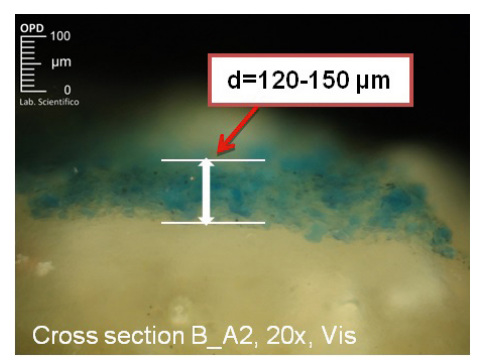

Fig. 12. Cross section B_A2, 20x, Vis (OPD).

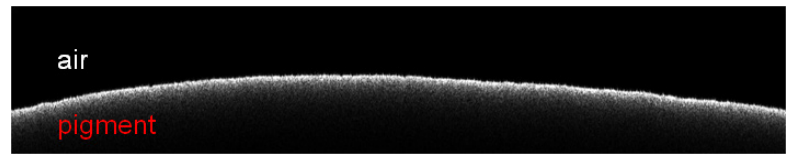

TdOCT profile@1550nm

$\mathrm{y}$ axis $=25 \mathrm{~mm}$

$\mathrm{z}$ axis $=1 \mathrm{~mm}$

Egg tempera layer thickness: $35-45 \mu \mathrm{m}$

Fig. 9. Sample R_A2 TdOCT profile $\left(\lambda_{\text {in }}=1550 \mathrm{~nm}\right)$.

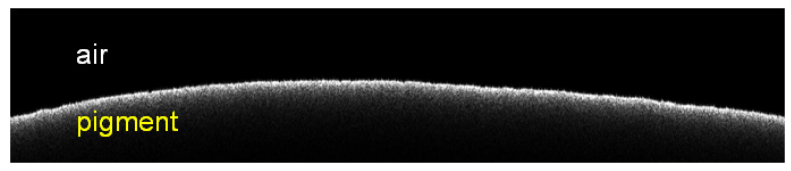

$$
\begin{aligned}
& \text { TdOCT profile @1550nm } \\
& \mathrm{y} \text { axis }=25 \mathrm{~mm} \\
& \mathrm{z} \text { axis }=1 \mathrm{~mm} \\
& \text { Egg tempera layer thickness: } 45-55 \mu \mathrm{m}
\end{aligned}
$$

Fig. 11. Sample Y_A2 TdOCT profile $\left(\boldsymbol{\lambda}_{\text {in }}=1550 \mathrm{~nm}\right)$.

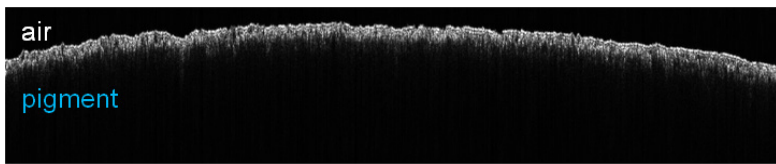

$$
\begin{aligned}
& \text { TdOCT profile @1550nm } \\
& \mathrm{y} \text { axis }=25 \mathrm{~mm} \\
& \mathrm{z} \text { axis }=1 \mathrm{~mm} \\
& \text { Egg tempera layer thickness: } 120-140 \mu \mathrm{m}
\end{aligned}
$$

Fig. 13. Sample B_A2 TdOCT profile $\left(\lambda_{\text {in }}=1550 \mathrm{~nm}\right)$.
All samples, especially the red and the yellow ones, show an intense and broad absorption band in the spectral detection range of non-linear microscopy, 400-600 $\mathrm{nm}$, thus suggesting the auto-absorption of the fluorescence radiation emitted as a consequence of the non-linear interaction inside the material. Within the spectral range of interest, minium and Cd-yellow show a similar behaviour: in both cases there is an absorption band between 350 and $550 \mathrm{~nm}$, and a following rapid increase of the reflectance values (probably due to the reflecting gypsum background), which reaches the $90 \%$ at $650 \mathrm{~nm}$ for minium and at $600 \mathrm{~nm}$ for yellow, remaining between 100\% and $80 \%$ up to $1700 \mathrm{~nm}$. With reference to the Egyptian blue, the reflectance spectrum 

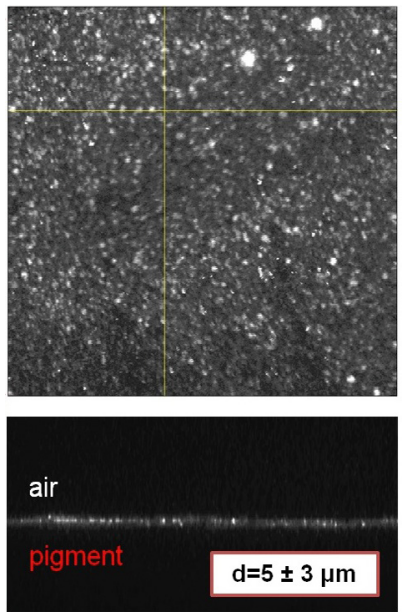

Fig. 14. Sample R_A2 grayscale TPEF intensity image (square 200 $\times 200 \mu \mathrm{m})$ and $\mathrm{z}$-stack profile $(\mathrm{z}=$ $100 \mu \mathrm{m})$.
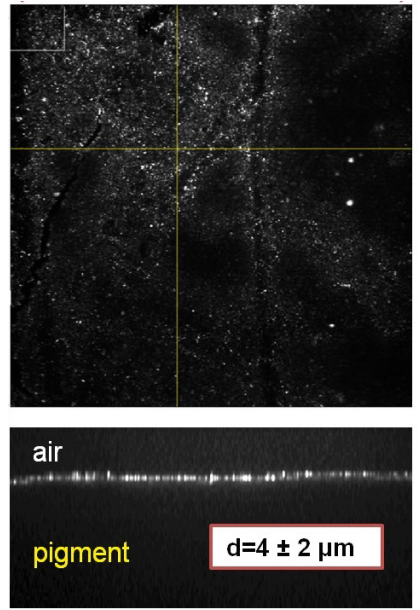

Fig. 15. Sample Y_A2 grayscale TPEF intensity image (square 200 $\times 200 \mu \mathrm{m})$ and $\mathrm{z}$-stack profile $(\mathrm{z}=$ $100 \mu \mathrm{m})$.
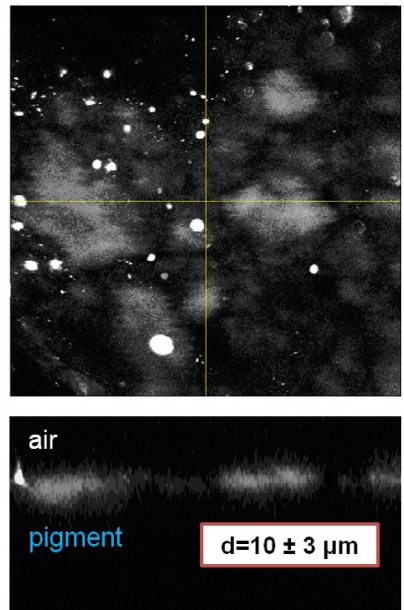

Fig. 16. Sample B_A2 grayscale TPEF intensity image (square 200 $\times 200 \mu \mathrm{m})$ and $\mathrm{z}$-stack profile $(\mathrm{z}=$ $100 \mu \mathrm{m})$.

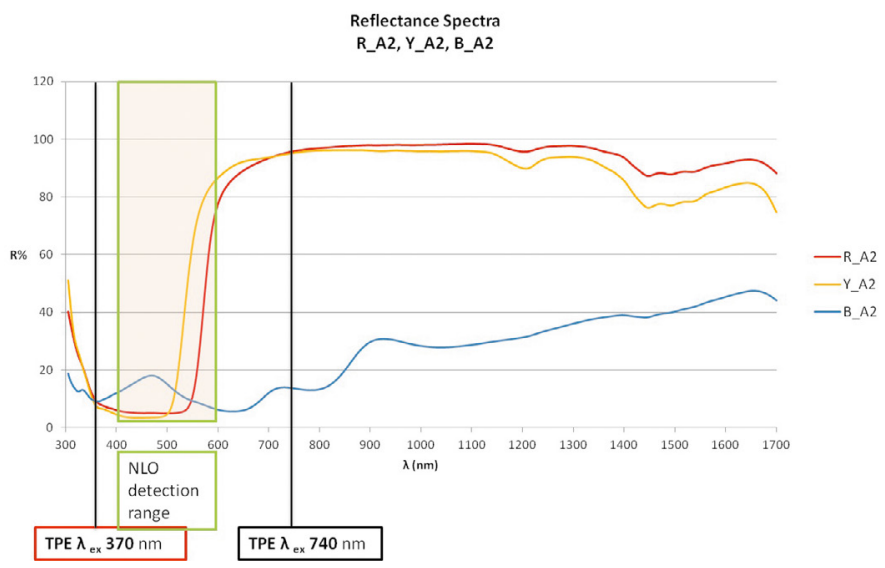

Fig. 17. Samples R_A2, Y_A2, B_A2 FORS Reflectance Spectra. The non-linear detection range (400-600 $\mathrm{nm}$ ) is highlighted in the green rectangle, as well as the TPEF excitation wavelength $(740 \mathrm{~nm})$. shows low reflectance at $740 \mathrm{~nm}$ (about 14\%) and $\mathrm{R}=40 \%$ at $1550 \mathrm{~nm}$. This behaviour is observed also in the images obtained with the Multispectral Vis-Nir Scanner (INO-CNR), shown in Fig. 18. Concerning the discrimination of the protective layers on the other samples, no significant results were found, except for samples R7, Y7, B7, which are covered with a protective layer made of white egg. The intensity fluorescence images (Fig. 19) show different micro-morphologies for the three pigments, which may be related to the drying process and the pigment particle size.

In any case, we were not able to evaluate the thickness of the superficial layers, or to discriminate the interfaces, probably because 

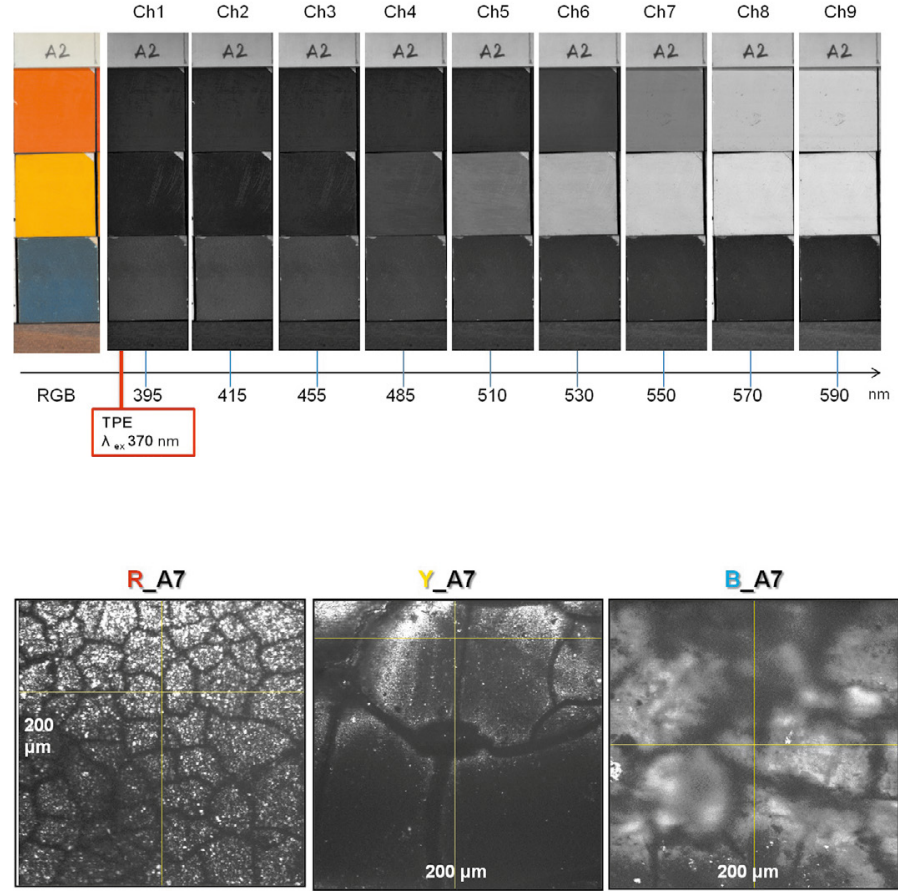

Fig. 19. Samples R_A7, Y_A7, B_A7 gray scale TPEF intensity image (square $200 \times$ $200 \mu \mathrm{m})$. of the transparency of the protective layers to the wavelength used. The discrimination of the three pigments was possible by means of FLIM by revealing different fluorescence lifetime, resulting in different trends in the decay curves. The evaluated average values of fluorescence lifetime for red lead, cadmium yellow and Egyptian blue are 240 ps, 170 ps, and over $30 \mathrm{~ns}$, respectively. It is worth noticing that the blue pigment fluorescence lifetime is well above the repetition rate of the laser (12 ns), thus resulting in a constantly linear trend of the decay curve (which is not reported). By contrast, the red and the yellow pigments (Fig. 20,21) are characterized by a significantly short fluorescence lifetime that is comparable or smaller than the acquisition time window of the system (which is approx. 200 ps). In conclusion, the FLIM analysis revealed relevant differences between the three pigments fluorescence lifetimes, especially for the blue pigment. The physi-

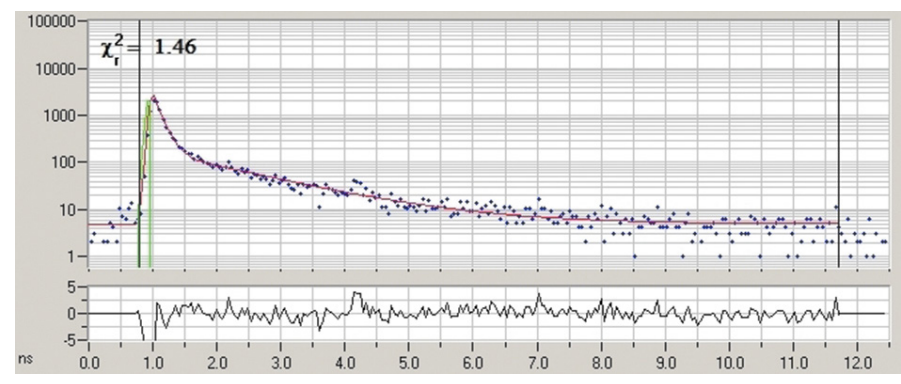

Fig. 20. Sample R_A2 Fluorescence lifetime decay curve. The time (ns) is reported on the $\mathrm{x}$ axis and the counts are reported on the $y$ axis. Recorded data (dots), monoexponential fit (red continuous line). 


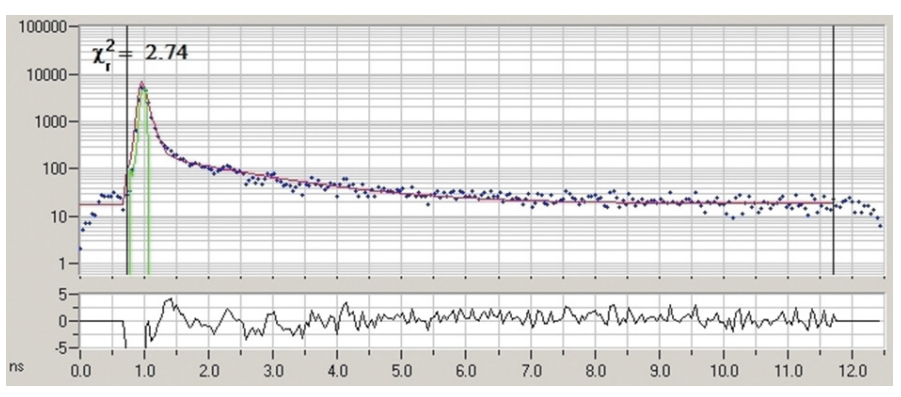

Fig. 21. Sample Y_A2 Fluorescence lifetime decay curve. The time (ns) is reported on the $\mathrm{x}$ axis and the counts are reported on the $y$ axis. Recorded data (dots), monoexponential fit (red continuous line). cal-chemical explanation of such different trends needs to be further investigated.

\section{Conclusions}

The purpose of this work is to describe whether and if so, to what extent NLO imaging techniques prove useful in probing multilayer painted objects, in evaluating the capability to collect non-linear signals and determine the layers thickness. In this respect, analyses were applied on a series of single- and multi-layers systems simulating real egg-tempera wood panel paintings. The preliminary results suggest that the $740 \mathrm{~nm}$ wavelength, while enabling the visualization of the painting surfaces micromorphology, is not effective for the determination of thickness through the detection of non-linear signals. On the basis of the combined analysis reported, we assume that a longer excitation wavelength may prove more effective. In collaboration with other research groups, further non-linear analyses with different devices and modalities are currently being conducted, as well as the linear fluorescence behaviour of each pigment is under investigation.

\section{Acknowledgements}

The research leading to these results was funded by the EU Community's H2020- Research Infrastructure programme under the IPERION CH Project (GA n. 654028), and in collaboration with Opificio delle Pietre Dure (Firenze).

\section{References}

[1] F. Faraldi, G.J. Tserevelakis, G. Filippidis, G.M. Ingo, C. Riccucci, C. Fotakis, "Multi photon excitation fluorescence imaging microscopy for the precise characterization of corrosion layers in silver-based artifacts" Applied Physics A, 111 (2013) 177-181, DOI: 10.1007/s00339-013-7548-z.

[2] G. Latour, J.P. Echard, M. Didier, M.C. SchanneKlein, "In situ 3D characterization of historical coatings and wood using multimodal nonlinear optical microscopy”, Optics Express, 20 (2012): 24623-24635, DOI: 10.1364/OE.20.024623.

[3] G. Filippidis, M. Massaouti, A. Selimis, E.J. Gualda, J.-M. Manceau, S. Tzortzakis, "Nonlinear imaging and $\mathrm{THz}$ diagnostic tools in the service of Cultural Heritage", Applied Physics A, 106 (2012), 257-263, DOI: 10.1007/s00339-014-8357-8.

[4] G. Filippidis, J. E. Gualda, K. Melessanaki, C. Fotakis, "Nonlinear imaging microscopy techniques as diagnostic tools for art conservation studies", Optics Letters 33 (2008) 240-242, DOI: 10.1364/ OL.33.000240.

[5] A. Nevin, D. Comelli, I. Osticioli, G. Filippidis, K. Melessanaki, G. Valentini, R. Cubeddu, C. Fotakis, "Multi-photon excitation fluorescence and third-harmonic generation microscopy measurements combined with confocal Raman microscopy for the analysis of layered samples of varnished oil films", Applied Physics A, 100 (2010) 599-606, DOI 10.1007/s00339-010-5644-x. 
[6] T. E. Villafana, W. Brown, W. S. Warren, and M. Fischer, "Ultrafast Pump-Probe Dynamics of Iron Oxide Based Earth Pigments for Applications to Ancient Pottery Manufacture", Proc. SPIE 9527, (2015) 952709, DOI: 10.1117/12.2184758.

[7] T.E. Villafana, P.W. Brown, J.K. Delaney, M. Palmerc, W. S. Warren, and M. C. Fischer, "Femtosecond pump-probe microscopy generates virtual cross-sections in historic artwork", Proc. of the National Academy of Sciences of the USA, 111 (2014) 1708-1713, DOI: $10.1073 /$ pnas.1317230111.

[8] G. Filippidis, K. Melessanaki and C. Fotakis, "Second and third harmonic generation measurements of glues used for lining textile supports of painted artworks", Analytical and Bioanalytical Chemistry (2009), DOI: 10.1007/s00216-009-3060-x.

[9] G. Filippidis, G. J. Tserevelakis, A. Selimis, C. Fotakis, "Nonlinear imaging techniques as non-destructive, high-resolution diagnostic tools for cultural heritage studies", Applied Physics A, 118 (2015) 417-423, DOI: 10.1007/s00339-014-8357-8.

[10] R. Mercatelli, F. Ratto, F. Rossi, F. Tatini, L. Menabuoni, A. Malandrini, R. Nicoletti, R. Pini, F. S. Pavone, R. Cicchi, “Three-dimensional mapping of the orientation of collagen corneal lamellae in healthy and keratoconic human corneas using SHG microscopy", Journal of Biophotonics, 10 (2017) 75-83, DOI: 10.1002/jbio.201600122.

[11] F. Rosi, C. Grazia, F. Gabrieli, A. Romani, M. Paolantoni, R. Vivani, B.G. Brunetti, P. Colomban, C. Miliani, "UV-Vis-NIR and micro Raman spectroscopies for the non destructive identification of $\mathrm{Cd}_{1-}$ ${ }_{\mathrm{x}} \mathrm{Zn}_{\mathrm{x}} \mathrm{S}$ solid solutions in cadmium yellow pigments", Microchemical Journal, 124 (2015) 856-867, DOI: 10.1016/j.microc.2015.07.025.

[12] G. Accorsi, A. Verri, M. Bolognesi, N. Armaroli, C. Clementi, C. Miliani, and A. Romani, "The exceptional near-infrared luminescence properties of cuprorivaite (Egyptian blue)", Chemical Communications (2009) 3392-3394, DOI: 10.1039/b902563d.

[13] P. Targowski, B. Rouba, M. Wojtkowski, A. Kowalczyk, "The application of optical coherence tomography to nondestructive examination of museum objects", Studies in Conservation, 49 (2004), 107-114.
[14] T. Arecchi, M. Bellini, C. Corsi, R. Fontana, M. Materazzi, L. Pezzati, A. Tortora, "A new tool for painting diagnostics: Optical coherence tomography" Optics and Spectroscopy 101 (2006), 23-26, DOI: 10.1134/S0030400X06070058.

[15] R. Fontana, M.C. Gambino, M. Greco, L. Marras, M. Materazzi, E. Pampaloni, L. Pezzati, "High precision surface analysis of roughness of Michelangelo's David", Proc. SPIE 5146 (2003) 236-243, DOI: 10.1117/12.501252.

[16] R. Fontana, M.C. Gambino, M. Greco, L. Marras, M. Materazzi, E. Pampaloni, L. Pezzati, P. Poggi, "Integrating 2D and 3D data for diagnostics of panel paintings”, Proc. SPIE 5146 (2003) 88-98, DOI: 10.1117/12.501247.

[17] R. Fontana, C. Mazzotta, M.C. Gambino, M. Greco, E. Pampaloni, L. Pezzati, "High-resolution 3D survey of artworks", Proc. SPIE 5457 (2004) 719-726, DOI: 10.1117/12.545660.

[18] R. Fontana, M. Barucci, E. Pampaloni, L. Pezzati, C. Daffara, "A study of surface optical properties for characterizing the cleaning process of paintings", Proc. SPIE 8790 (2013) 87900O, DOI: 10.1117/12.2020738.

[19] C. Bonifazzi, P. Carcagni, R. Fontana, M. Greco, M. Mastroianni, M. Materazzi, E. Pampaloni, L. Pezzati, D. Bencini, "A scanning device for VIS-NIR multispectral imaging of paintings", Journal of Optics A: Pure and Applied Optics, 10 (2008), 064011 , DOI: 10.1088/1464-4258/10/6/064011.

[20] P. Carcagni, Patria A. Della, R. Fontana, M. Greco, M. Mastroianni, M. Materazzi, E. Pampaloni, L. Pezzati, "Multispectral imaging of paintings by optical scanning", Optics and Lasers in Engineering, 45 (2007), 360-367, DOI: 10.1016/j. optlaseng.2005.02.010.

[21] R. Fontana, M. Barucci, E. Pampaloni, J. Striova, L. Pezzati, "From Leonardo to Raffaello: insights by Vis-IR Reflectography”, in: Acta Artis Academica 2014 Proceedings of the $5^{\text {th }}$ interdisciplinary Conference of ALMA, Interpretation of Fine Art's Analysis in Diverse Contexts, D. Hradil, J. Hradilová (Eds.), Academy of Fine Arts in Prague, Prague 2014, pp.: 15-26, ISBN 978-80-87108-48-2. 
